\title{
Identidad y distribución de Bouteloua reederorum (Poaceae: Chloridoideae), una especie endémica de México
}

\section{Identity and distribution of Bouteloua reederorum (Poaceae: Chloridoideae), a species endemic to Mexico}

\section{Acta Botanica Mexicana}

\author{
Manuel Higinio Sandoval-Ortega'1/4 (iD, María Elena Siqueiros-Delgado' (iD, Rosa Cerros-Tlatilpa² (iD), Eugenio Pérez-Molphe Balch³ (iD
}

\begin{abstract}
Resumen:
Antecedentes y Objetivos: Bouteloua es uno de los géneros más importantes en los pastizales de Norteamérica, especialmente en México, donde se distribuye $92 \%$ de los taxones descritos para este grupo, 25 de los cuales son endémicos. Uno de estos es Bouteloua reederorum, que ha sido confundido con $B$. stolonifera, ocasionando que sea reportado en estados de la república en donde no se encuentra. El objetivo del presente trabajo es aclarar la identidad de B. reederorum y proporcionar una descripción morfológica detallada con fotografías de estructuras importantes para su identificación, así como un mapa de distribución para esta especie.

Métodos: Se revisó el material depositado en los herbarios ARIZ, HUAA, HUAP, HUMO, IEB, INEGI, MEXU, XAL y las colecciones digitales MO, P y US y la plataforma JSTOR, para obtener registros de $B$. reederorum. Con base en esta información se determinaron los puntos de colecta, que fueron visitados de septiembre de 2017 a agosto de 2019 en busca de poblaciones de esta especie, donde se colectaron ejemplares. El material colectado fue identificado y revisado para elaborar una descripción morfológica con fotografías de características importantes para la identificación de la especie. Con los datos tomados en campo, material de herbario y colecciones digitales se realizó un mapa de distribución de $B$. reederorum.

Resultados clave: Bouteloua reederorum y $B$. stolonifera pueden distinguirse fácilmente por caracteres morfológicos como la ramificación de sus inflorescencias y la forma de la lígula. Bouteloua reederorum se encuentra restringida al Valle de Tehuacán-Cuicatlán, ubicado entre Puebla y Oaxaca. Aunque generalmente las espiguillas masculinas de $B$. reederorum no tienen flósculos rudimentarios, en raras ocasiones pueden desarrollar uno, similar al de las espiguillas femeninas.
\end{abstract}

Conclusiones: Bouteloua reederorum es distinta de Bouteloua stolonifera (=Cyclostachya stolonifera). Es necesario realizar estudios fenológicos para determinar si B. reedeorum puede ser dioica.

Palabras clave: Cyclostachya stolonifera, nomenclatura, Pringleochloa stolonifera, taxonomía, Valle de Tehuacán-Cuicatlán.

\section{Abstract:}

Background and Aims: Bouteloua is one of the most important genera in North American grasslands, especially in Mexico, where $92 \%$ of the taxa described for this group are distributed, 25 of which are endemic. One of these is Bouteloua reederorum, which has been confused with $B$. stolonifera, causing it to be reported in states of the republic where it is not found. The aim of the present work is to clarify the identity of $B$. reederorum and provide a detailed morphological description with photographs of important structures for its identification, as well as a distribution map for this species.

Methods: The material deposited in the herbaria ARIZ, HUAA, HUAP, HUMO, IEB, INEGI, MEXU, XAL, the digital collections MO, P and US, and the platform JSTOR, were reviewed to obtain records of $B$. reederorum. Based on this information, collection points were determined, which were visited from September 2017 to August 2019 in search of populations of this species, where specimens were collected. The collected material was identified and reviewed to prepare a morphological description accompanied with photographs of important characteristics for the identification of the species. With the obtained records, a distribution map of $B$. reederorum was made.

Key results: Bouteloua reederorum and $B$. stolonifera can be easily distinguished by several morphological characteristics such as branching of their inflorescences and the ligule shape. Bouteloua reederorum is practically restricted to the Tehuacán-Cuicatlán Valley, located between Puebla and Oaxaca. Although the male spikelets of $B$. reederorum generally do not have rudimentary florets, they can rarely develop one similar to that of the female spikelets.

Conclusions: Bouteloua reederorum is different from Bouteloua stolonifera (= Cyclostachya stolonifera). Phenological studies are necessary to determine if $B$. reedeorum can be dioecious.

Key words: Cyclostachya stolonifera, nomenclature, Pringleochloa stolonifera, taxonomy, Tehuacán-Cuicatlán Valley.

${ }^{1}$ Universidad Autónoma de Aguascalientes, Herbario HUAA, Centro de Ciencias Básicas, Departamento de Biología, Av. Universidad 940, Ciudad Universitaria, 20131 Aguascalientes, Aguascalientes, México.

2Universidad Autónoma del Estado de Morelos, Facultad de Ciencias Biológicas, Laboratorio de Sistemática y Morfología, Av. Universidad 1001, Col. Chamilpa, 62210 Cuernavaca, Morelos, México.

${ }^{3}$ Universidad Autónoma de Aguascalientes, Centro de Ciencias Básicas, Departamento de Química, Laboratorio de Biología Molecular, Avenida Universidad 940, Ciudad Universitaria, 20131 Aguascalientes, Aguascalientes, México.

${ }^{4}$ Autor para la correspondencia: m.higinio.s@hotmail.com
Recibido: 18 de marzo de 2020

Revisado: 3 de abril de 2020

Aceptado por Rosario Redonda Martínez: 12 de agosto de 2020.

Publicado Primero en línea: 9 de septiembre de 2020.

Publicado: Acta Botanica Mexicana 128 (2021).
Citar como: Sandoval-Ortega, M. H., M. E. Siqueiros-Delgado, R. Cerros-Tlatilpa y E. Pérez-Molphe Balch. 2020(2021). Notas sobre la identidad y distribución de Bouteloua reederorum (Poaceae: Chloridoideae), una especie endémica de México. Acta Botanica Mexicana 128: e1698. DOI: https://doi.org/10.21829/abm128.2021.1698 


\section{Introducción}

Bouteloua Lag. es un género integrado por 60 especies y uno de los más importantes en los pastizales de Norteamérica, debido a que es un componente primario en estas comunidades e incluye especies de alto valor forrajero (Gould, 1979; Herrera-Arrieta et al., 2004; Peterson et al., 2015). En México se distribuye $92 \%$ de los taxones descritos para este grupo, 25 de los cuales son endémicos, por lo que se ubica como uno de los cinco géneros de pastos con mayor riqueza en el país (Herrera-Arrieta et al., 2004; Sánchez-Ken, 2019).

Con 13 especies diclinas, Bouteloua presenta amplia variación en la distribución de sexos (Kinney et al., 2007). Históricamente, los taxónomos han segregado a los pastos con flores unisexuales de sus parientes hermafroditas, colocándolos en grupos distintos (Columbus, 1999), de tal modo que todos los integrantes de Bouteloua que presentan flores unisexuales se clasificaban en los géneros Buchloe Engelm., Buchlomimus Reeder, C. Reeder, \& Rzed., Cathestecum J. Presl, Cyclostachya Reeder \& C. Reeder, Griffithsochloa G.J. Pierce, Opizia J. Presl, Pringleochloa Scribn. y Soderstromia C.V. Morton (Reeder y Reeder, 1963; Reeder et al., 1965; Reeder, 1969; Columbus et al., 1998; Columbus, 1999; Kinney et al., 2007). Bouteloua reederorum Columbus, endémica de México, es un ejemplo de esto, debido a que era considerada el único representante de Pringleochloa (Scribner, 1896; Columbus, 1999).

Aun cuando existen registros de que $B$. reederorum se distribuye exclusivamente en los estados de Puebla y Oaxaca (Scribner, 1896; Columbus et al., 1998; Beetle et al., 1999; Espejo-Serna y López-Ferrari, 2000), se ha reportado también para Aguascalientes, Durango, San Luis Potosí y Zacatecas (Herrera-Arrieta et al., 2010; Herrera-Arrieta, 2014; Villaseñor, 2016; Sánchez-Ken, 2019). Es probable que lo anterior se deba a que Cyclostachya stolonifera (Scribn.) Reeder \& C. Reeder ha sido considerado erróneamente como un sinónimo de B. reederorum (Herrera-Arrieta et al., 2010; Peterson et al., 2001), cuando en realidad C. stolonifera es sinónimo de B. stolonifera Scribn. (Columbus, 1999), la cual es dioica y se distribuye en el norte del país. Debido a la confusión existente entre estos dos taxones, los ejemplares de $B$. stolonifera son identificados como $B$. reederorum, lo que genera falsos reportes de esta última en sitios en los que en realidad no se encuentra.
El objetivo del presente trabajo es aclarar la identidad taxonómica de $B$. reederorum y $B$. stolonifera, determinando los sinónimos correctos por medio de una revisión nomenclatural, así como los caracteres que distinguen a $B$. reederorum de $B$. stolonifera (= Cyclostachya stolonifera) y proporcionar una descripción morfológica detallada de $B$. reederorum, acompañada con fotografías de estructuras importantes para su identificación, además de un mapa de distribución para esta especie.

\section{Materiales y Métodos}

Se revisó el material identificado como $B$. reederorum en los herbarios: ARIZ (Universidad de Arizona), HUAP (Benemérita Universidad Autónoma de Puebla), HUAA (Universidad Autónoma de Aguascalientes), HUMO (Universidad Autónoma del Estado de Morelos), IEB (Instituto de Ecología, A.C., Centro Regional del Bajío), INEGI (Instituto Nacional de Estadística y Geografía), MEXU (Herbario Nacional de México, Instituto de Biología, Universidad Nacional Autónoma de México) y XAL (Instituto de Ecología, A.C., Xalapa), así como las colecciones digitales MO (Missouri Botanical Garden (TROPICOS, 2020)), US (Smithsonian Institution (US, 2019)), P (Muséum national d'Histoire naturelle, París (P, 2019)) y la plataforma Global Plants (JSTOR, 2019), los acrónimos están citados de acuerdo con el Index Herbariorum (Thiers, 2019+).

Con base en la información obtenida en las colecciones digitales y del material de los herbarios revisados, se determinaron las localidades de colecta en los municipios Atoyatempan, Cuapiaxtla de Madero, Quecholac, San Sebastián Zinacatepec, Tecamachalco, Tehuacán, Tepeyahualco de Cuauhtémoc en el estado de Puebla y Santiago Chazumba, en el distrito Huajuapan, del estado de Oaxaca, las cuales fueron visitadas de septiembre de 2017 a agosto de 2019. En cada sitio se tomaron coordenadas geográficas utilizando un GPS eTrex ${ }^{\circledR}$ 20x (Garmin International Inc., Kansas City, EUA), con base en Datum WGS 84 (DMA, 1991). Los ejemplares colectados durante el trabajo de campo en Puebla y Oaxaca están incluidos en la lista de ejemplares examinados.

Los ejemplares se colectaron con hasta cuatro duplicados, utilizando la metodología propuesta por Engelmann (1986). El material se identificó con bibliografía especializada (Scribner, 1896; Beetle et al., 1999), se cotejó con material herborizado y con imágenes digitales de los ejemplares cita- 
dos por Scribner (1896), así como del tipo de Atheropogon stolonifer E. Fourn. (basónimo de B. reederorum) depositado en el herbario de la Universidad de Copenhague (C) (Sandoval-Ortega et al., 2019). Los especímenes de B. reederorum fueron depositados en el herbario HUAA y se enviaron duplicados a HUAP, CIIDIR (Centro Interdisciplinario de Investigación para el Desarrollo Integral Regional, Instituto Politécnico Nacional) y MEXU.

Utilizando información bibliográfica (Reeder y Reeder, 1963; de la Cerda-Lemus, 1996; Herrera-Arrieta, 2001; Dávila et al., 2018), material colectado y el examinado en herbarios se realizó un cuadro comparativo para diferenciar $B$. reederorum de $B$. stolonifera, la revisión nomenclatural y una descripción morfológica de $B$. reederorum con base en la secuencia de órganos utilizada por Herrera-Arrieta y Peterson (2018). Se tomaron fotografías con un microscopio estereoscópico Leica EZ4 E (Leica Microsystems, Wetzlar, Alemania) y una cámara fotográfica SONY $\alpha$ 7II (SONY corporation, Tokyo, Japón), de estructuras para facilitar su identificación. Se elaboró un mapa de distribución de $B$. reederorum con el programa QGIS v. 2.28.4 (QGIS Development Team, 2017), utilizando los datos de material recolectado, especímenes de herbario y colecciones digitales.

\section{Resultados}

Bouteloua reederorum y $B$. stolonifera son dos especies diferentes que pueden distinguirse fácilmente por caracteres morfológicos como la ramificación de sus inflorescencias y la forma de la lígula (Cuadro 1). Bouteloua stolonifera es uno de los dos taxones estrictamente dioicos del género Bouteloua, que se caracteriza principalmente por sus inflorescencias con una sola rama que, en la madurez, se curva y llegan a formar un círculo (Fig. 1); mientras que $B$. reederorum es una especie monoica con inflorescencias ramificadas que no forman un círculo en la madurez.

\section{Taxonomía}

Bouteloua reederorum Columbus, Aliso 18(1): 63. 1999. Fig. 2.

इAtheropogon stolonifer E. Fourn. Mexic. PI. 2: 140. 1886. TIPO: MÉXICO. Oaxaca, La Parada, Sierra de Oaxaca, 2590 m, IV.1842, F. M. Liebmann 588 (holotipo: C-10022902!, isotipo: US-00156937!, fragmento).

三 Pringleochloa stolonifera (E. Fourn.) Scribn., Bot. Gaz. 21(3): 138. 1896.

Plantas herbáceas, perennes, monoicas, amacolladas, estoloníferas; tallos con inflorescencias masculinas erectos o geniculados, $10-26 \mathrm{~cm}$ de largo, los de las inflorescencias femeninas horizontales o ascendentes, mucho más cortos, 2-5 cm de largo, nudos de color amarillo o marrón, puberulentos a glabrescentes, entrenudos glabros y estriados; hojas dispuestas en la base del macollo, vainas glabras a esparcidamente pubescentes con tricomas simples blanquecinas, márgenes hialinos, más cortas que los entrenudos en los tallos con inflorescencias masculinas y

Cuadro 1: Diferencias entre Bouteloua reederorum Columbus y B. stolonifera Scribn. *Fuentes: Reeder y Reeder (1963); de la Cerda-Lemus (1996); Herrera-Arrieta (2001); Dávila et al. (2018).

\begin{tabular}{llc}
\hline Caracter & $\begin{array}{c}\text { Bouteloua reederorum Columbus (= Pringleochloa } \\
\text { stolonifera (E. Fourn.) Scribn.) }\end{array}$ & $\begin{array}{c}\text { Bouteloua stolonifera Scribn. (= Cyclostachya stolonifera } \\
\text { (Scribn.) Reeder \& C. Reeder) }\end{array}$ \\
\hline $\begin{array}{l}\text { Distribución de sexos } \\
\text { Distribución geográfica }\end{array}$ & Monoica & Dioica \\
& Puebla, Oaxaca & Aguascalientes, Ciudad de México, Durango, Hidalgo, \\
Lígula & Tricomas & Membranosa, hialina \\
Inflorescencias masculinas & Ramificada, sin curvarse en la madurez & No ramificada, curvándose en la madurez \\
Inflorescencias femeninas & Ramificadas, en la base del macollo, sin curvarse en la la & No ramificadas, por encima del macollo, curvándose en \\
& madurez & la madurez \\
Espiguillas masculinas & 1 flósculo fértil y 1 flósculo rudimentario & 1 flósculo fértil, flósculo rudimentario ausente \\
Espiguillas femeninas & 3-8(11) en cada rama & $\geq 15$ en cada rama \\
\hline
\end{tabular}



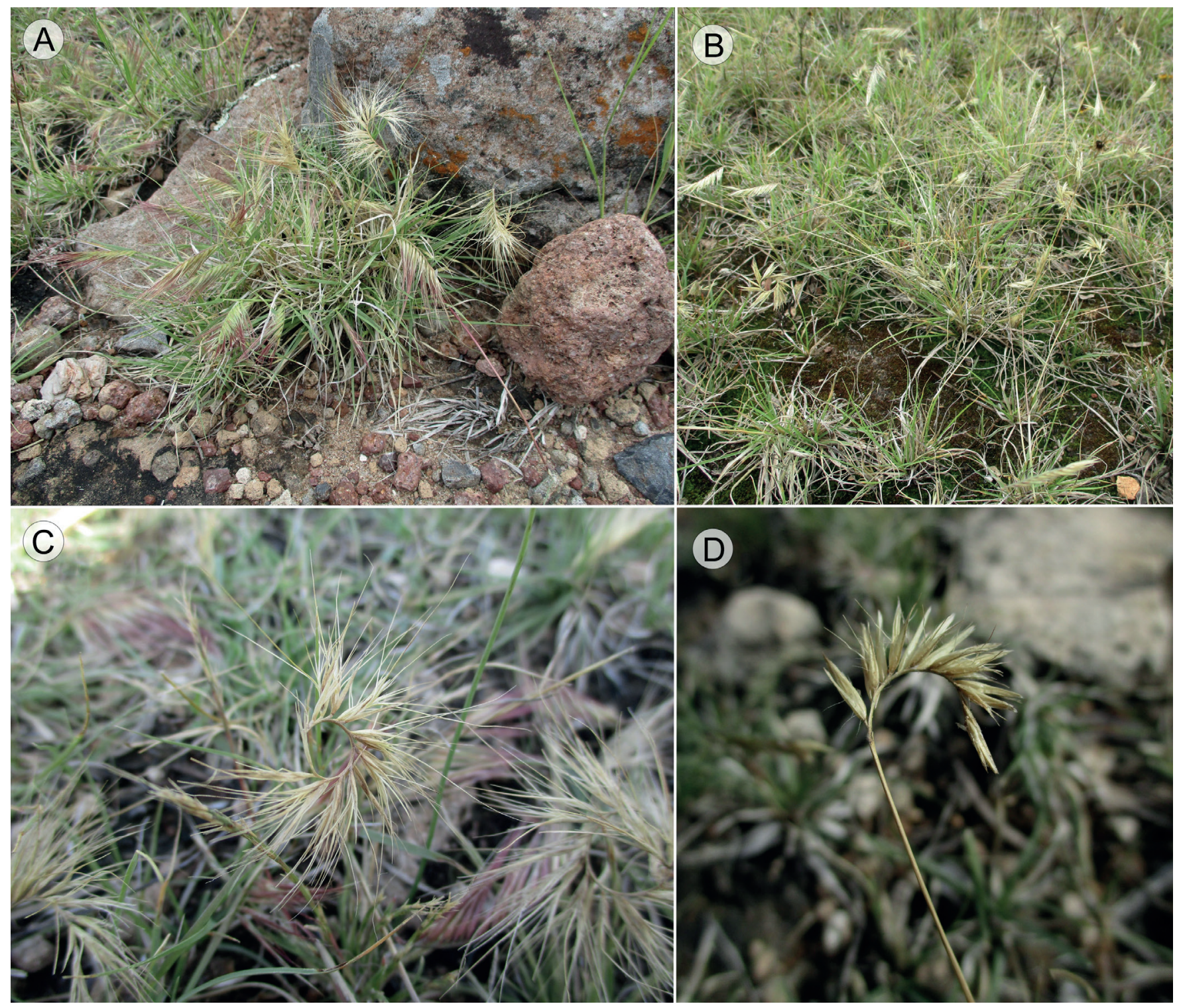

Figura 1: Bouteloua stolonifera Scribn. A. planta femenina; B. planta masculina; C. inflorescencia femenina; D. inflorescencia masculina.

más largas que los entrenudos en los tallos con inflorescencias femeninas, láminas 2-11 cm de largo, planas, glabras a esparcidamente puberulentas con tricomas simples blanquecinos, margen involuto al secarse, lígulas de tricomas, éstos ca. $1 \mathrm{~mm}$ de longitud; inflorescencias masculinas erguidas por encima del macollo, ramificadas, raquis 1.5$3(3.5) \mathrm{cm}$ de largo, distalmente aplanado, puberulento, ramas 5-6, pectinadas (Figs. 2B, 3A), ascendentes a horizontales, raquillas $(0.4) 1-1.5(1.8) \mathrm{cm}$ de largo, aplanadas, puberulentas, con un mechón de tricomas más largos cerca de la base y por debajo de cada espiguilla, ramas con (7)1015(18) espiguillas, glumas subiguales (Fig. 3D), primera gluma linear-lanceolada, 2.7-3.2(3.5) $\mathrm{mm}$ de largo, hialina, pubescente sobre la vena media con tricomas similares a los de la raquilla, uninervada, ápice acuminado, aristado (Fig. 3E), segunda gluma ovado elíptica, 4.2-5 mm de largo, color morado, glabra a esparcidamente puberulenta en la base, uninervada, ápice con un mucrón en medio de dos lóbulos membranosos ápice agudo ligeramente más corto (Fig. 3F), flósculo fértil 1, flósculos rudimentarios ausentes (Figs. 3B, C); inflorescencias femeninas restringidas a la base del macollo (Figs. 2C, 4A), raquis aplanado, 0.7-1.2 cm de largo, viloso, ramas 4-5, compactas, semejando glomérulos, ascendentes, raquillas 3-5 $\mathrm{mm}$ de largo, puberulen- 


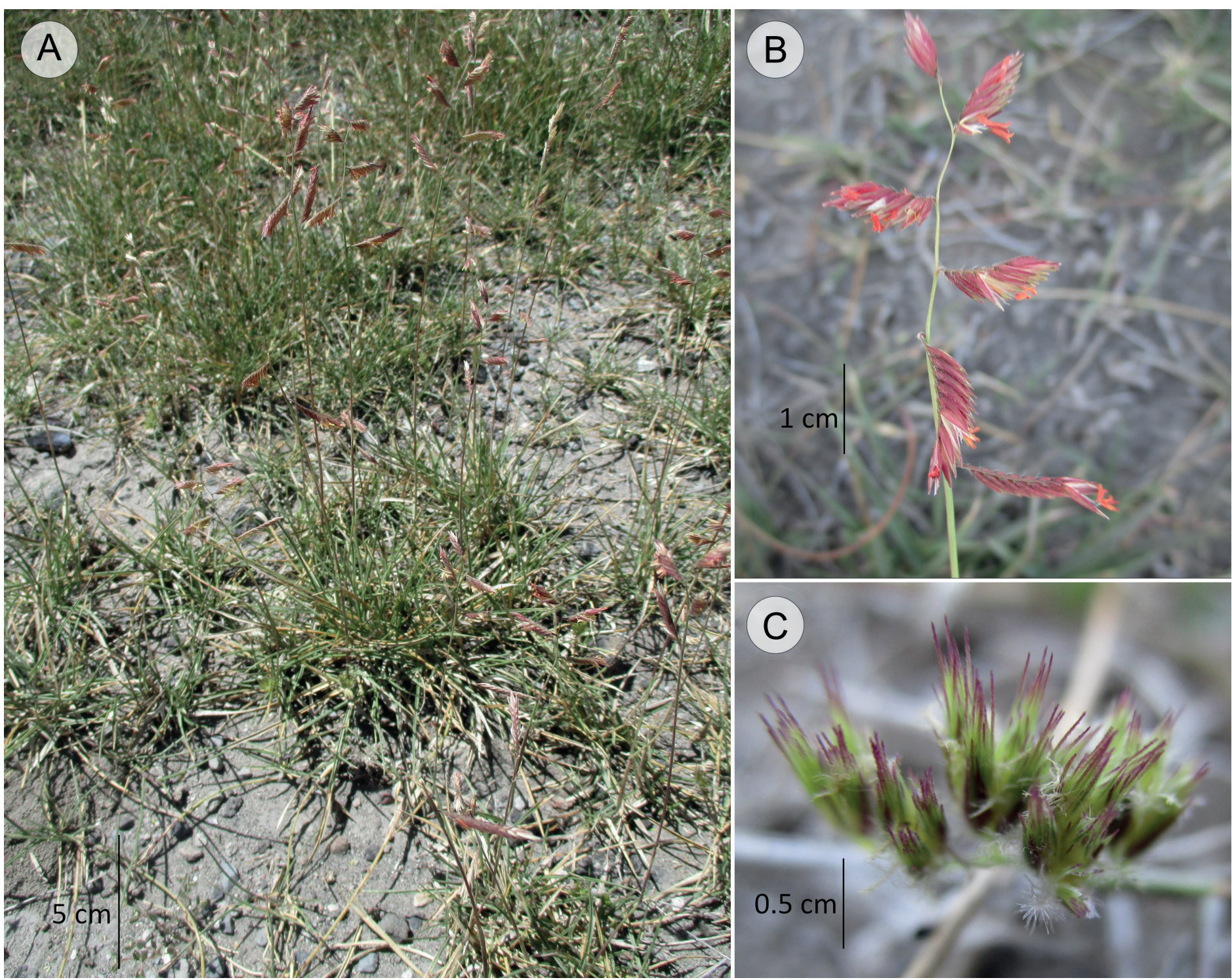

Figura 2: Bouteloua reederorum Columbus. A. vista general; B. inflorescencia masculina; C. inflorescencia femenina.

tas, ramas con 3-8(11) espiguillas, glumas subiguales (Fig. 4D), primera gluma 2.8-3(4) $\mathrm{mm}$ de largo, linear-lanceolada, hialina, uninervada (Fig. 4E), segunda gluma (4.5)5-5.3 $\mathrm{mm}$ de largo, ovado-lanceolada, color verde-amarillenta a morada, uninervada, ápice atenuado, en ocasiones con un par de lóbulos membranosos cortos, vena media proyectándose a manera de arista escabrosa (Fig. 4F), flósculo fértil 1, flósculos rudimentarios 1-2 (Figs. 4B, C); flósculo estaminado con lema ovado-elíptica, 3.5-4(4.3) mm de largo, hialino-amarillenta, glabra, trinervada, ápice agudo, 3-aristado (Fig. 3G), pálea ovado-lanceolada, 3.5-4 mm de largo, hialina, glabra, 2-nervada, ápice agudo, 2-mucronado (Fig. $3 \mathrm{H})$; flósculo pistilado con lema ovado-elíptica, 5.5-6 mm de largo, hialino-amarillenta, pubescente, 3-nervada, venas laterales extendiéndose subapicalmente a manera de aristas escabrosas, ápice corto aristado (Fig. 4G), pálea casi tan larga como la lema, ovado lanceolada, hialina, distalmente pubescente, 2-nervada, ápice agudo, 2-mucronado (Fig. $4 \mathrm{H}$ ); ovario ca. $1.3 \mathrm{~mm}$ de largo, obovado, estigmas plumosos (Fig. 4I); flósculos rudimentarios con lema y pálea flabeliformes, 5-6(7) mm de largo, con numerosas aristas escabrosas, moradas en el ápice (Fig. 4J).

Notas taxonómicas: aunque se describen las espiguillas masculinas de $B$. reederorum como desprovistas de flósculos rudimentarios, se observó la presencia de estas 


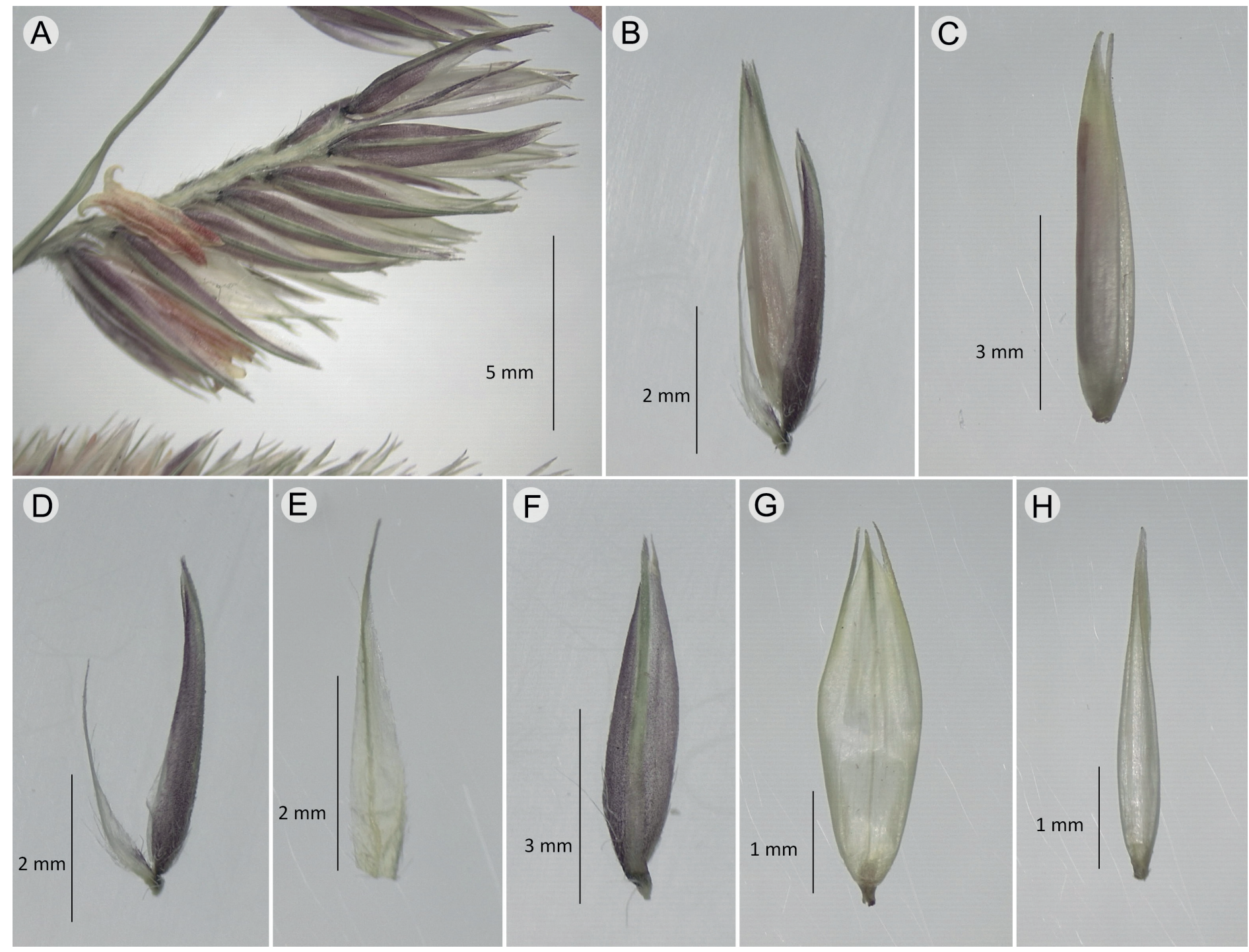

Figura 3: Inflorescencia masculina y estructuras de espiguilla estaminada de Bouteloua reederorum Columbus. A. rama de la inflorescencia; B. espiguilla; C. flósculo fértil; D. glumas vacías; E. primera gluma; F. segunda gluma; G. lema; H. pálea.

estructuras en espiguillas de las ramas apicales en un par de ejemplares (Fig. 5). En ambos casos, el flósculo que debería ser fértil, con lema y pálea bien desarrolladas, carecía de estambres. Los flósculos rudimentarios observados en estas espiguillas son flabeliformes y con múltiples aristas (Figs. 5C, D), como sucede en las espiguillas femeninas, pero de menor tamaño, $\leq 2 \mathrm{~mm}$ de largo.

Distribución: endémica de México, en los estados de Puebla y Oaxaca, se encuentra restringida al Valle de Tehuacán-Cuicatlán (Fig. 6), en elevaciones de 1100 a 2400 m s.n.m.
Hábitat: matorral xerófilo y pastizal, principalmente en planicies con suelo arenoso y calcáreo, donde llega a ser predominante (Fig. 7). También se puede encontrar en áreas con disturbio como orilla de caminos y campos de cultivo abandonados, junto con otras especies del mismo género como Bouteloua dimorpha Columbus y B. repens (Kunth) Scribn. \& Merr.

Fenología: florece y fructifica de agosto a octubre.

Ejemplares examinados: MÉXICO. Puebla, municipio Atoyatempan, zona jardín de Los Saberes, 1893 m, $18^{\circ} 46^{\prime} 43^{\prime \prime} \mathrm{N}, 97^{\circ} 54^{\prime} 37^{\prime \prime} \mathrm{W}, 6 . X .2011$, L. C. Onofre 532 (HUAP); 

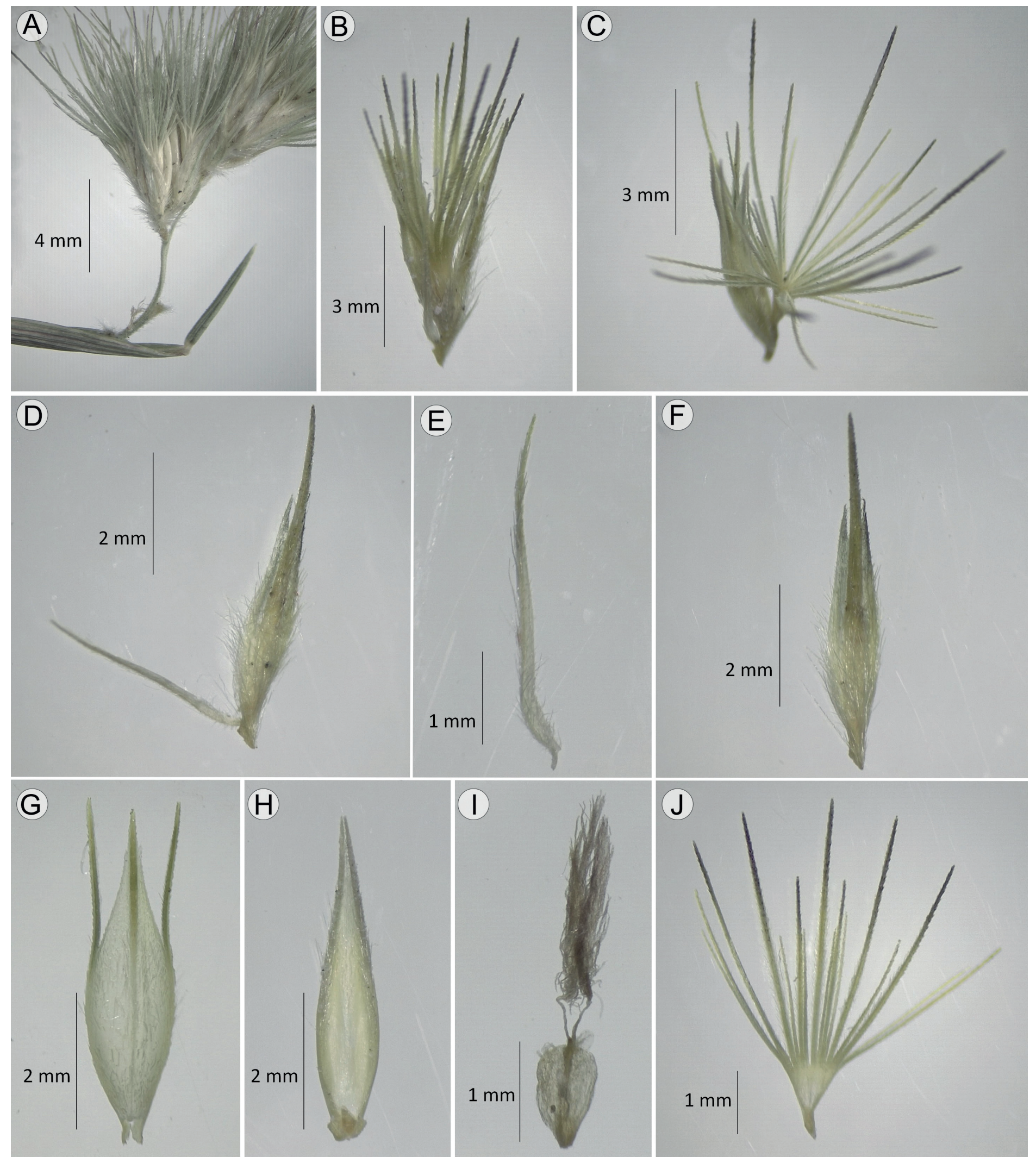

Figura 4: Inflorescencia femenina y estructuras de espiguilla pistilada de Bouteloua reederorum Columbus. A. rama de la inflorescencia con base del raquis desnudo; B. espiguilla; C. glumas; D. flósculo fértil y rudimentario sin glumas; E. primera gluma; F. segunda gluma; G. lema; H. pálea; I. ovario; J. lema del primer flósculo rudimentario. 


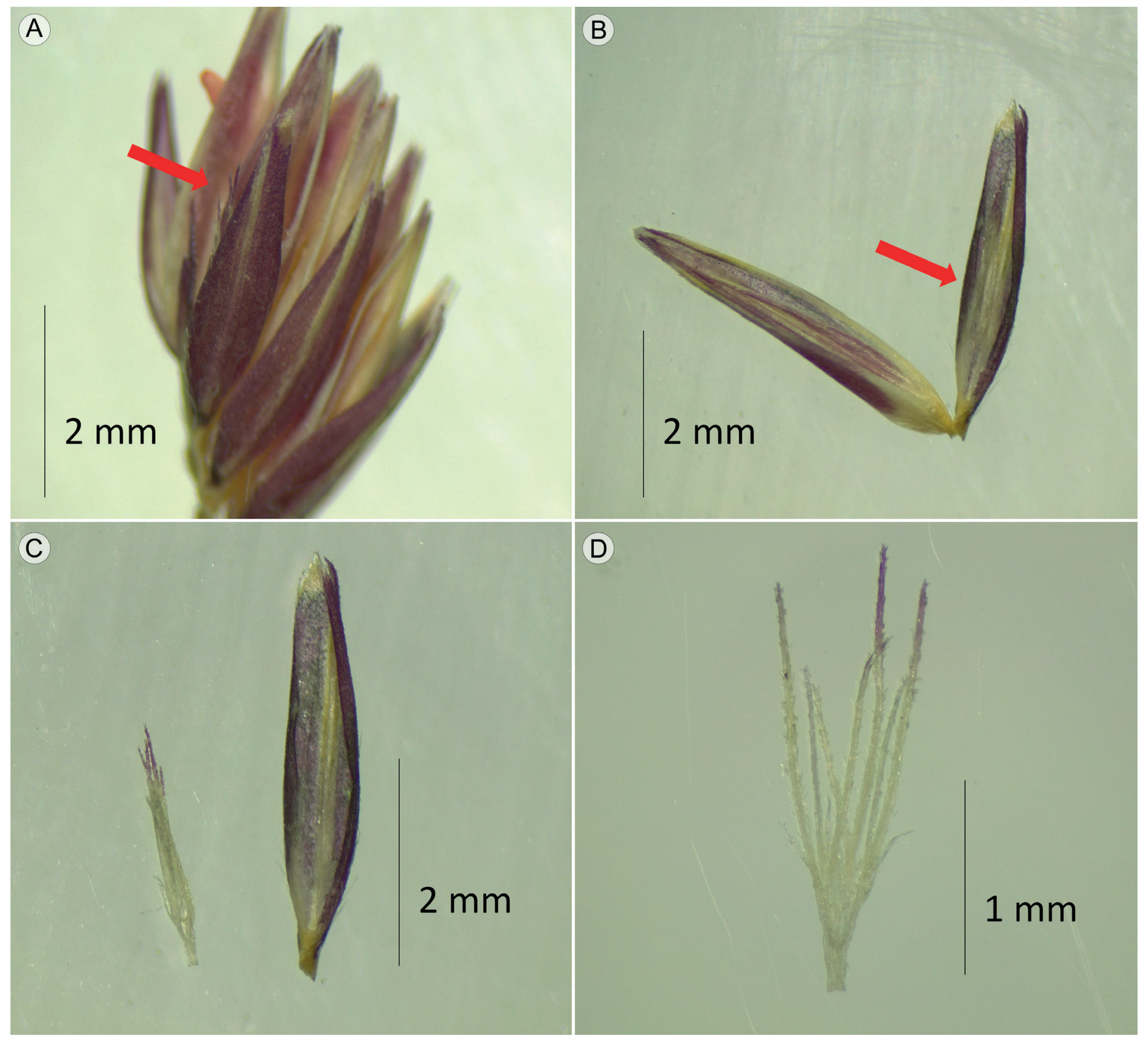

Figura 5: Flósculo rudimentario en espiguilla masculina de Bouteloua reederorum Columbus. A. rama de la inflorescencia y espiguilla con flósculo rudimentario señalado con flecha roja; B. flósculo rudimentario sobre segunda gluma señalado con flecha roja y flósculo estéril con lema y pálea bien desarrolladas; C. flósculo rudimentario y segunda gluma; D. detalle de flósculo rudimentario.

$2.3 \mathrm{~km}$ al SE de Atoyatempan, $1939 \mathrm{~m}, 18^{\circ} 47^{\prime} 43.8^{\prime \prime} \mathrm{N}$, 9754'42.7"W, 6.X.2018, M. H. Sandoval 1032 (HUAA). Municipio Cuapiaxtla de Madero, Hermosillo, extremo SE de Cuapiaxtla de Madero, 2039 m, 18 $54^{\prime} 22.5^{\prime \prime} \mathrm{N}$, $97^{\circ} 48^{\prime} 57.1^{\prime \prime W}, 6 . X .2018$, M. H. Sandoval 1030 (HUAA). Municipio Molcaxac, 34 mi SE of Puebla, 2164 m, 12.IX.1963, J. R. Reeder y C. G. Reeder 3739 (ARIZ). Municipio Quecholac, a 2 km al NO de San José Tuzuapan, carretera Tecama- chalco - Quecholac, $2143 \mathrm{~m}, 18^{\circ} 54^{\prime} 46.9^{\prime \prime} \mathrm{N}, 97^{\circ} 41^{\prime} 36.1^{\prime \prime} \mathrm{W}$, 6.X.2018, M. H. Sandoval 1029 (HUAA). Municipio San Sebastián Zinacatepec, orilla SE de San Sebastián Zinacatepec, $1138 \mathrm{~m}, 18^{\circ} 19^{\prime} 30.6^{\prime \prime} \mathrm{N}, 9^{\circ} 15^{\prime} 15.6^{\prime \prime} \mathrm{W}, 7 . X .2018$, M. H. Sandoval 1035 (HUAA). Municipio Tecamachalco, 31 mi NW of Tehuacán, 2010 m, 12.IX.1963, J. R. Reeder y C. G. Reeder 3733 (ARIZ); 8 mi SE of Acatzingo, 2438 m, 14.VIII.1965, J. R. Reeder y C. G. Reeder 4396 (ARIZ, MEXU); 


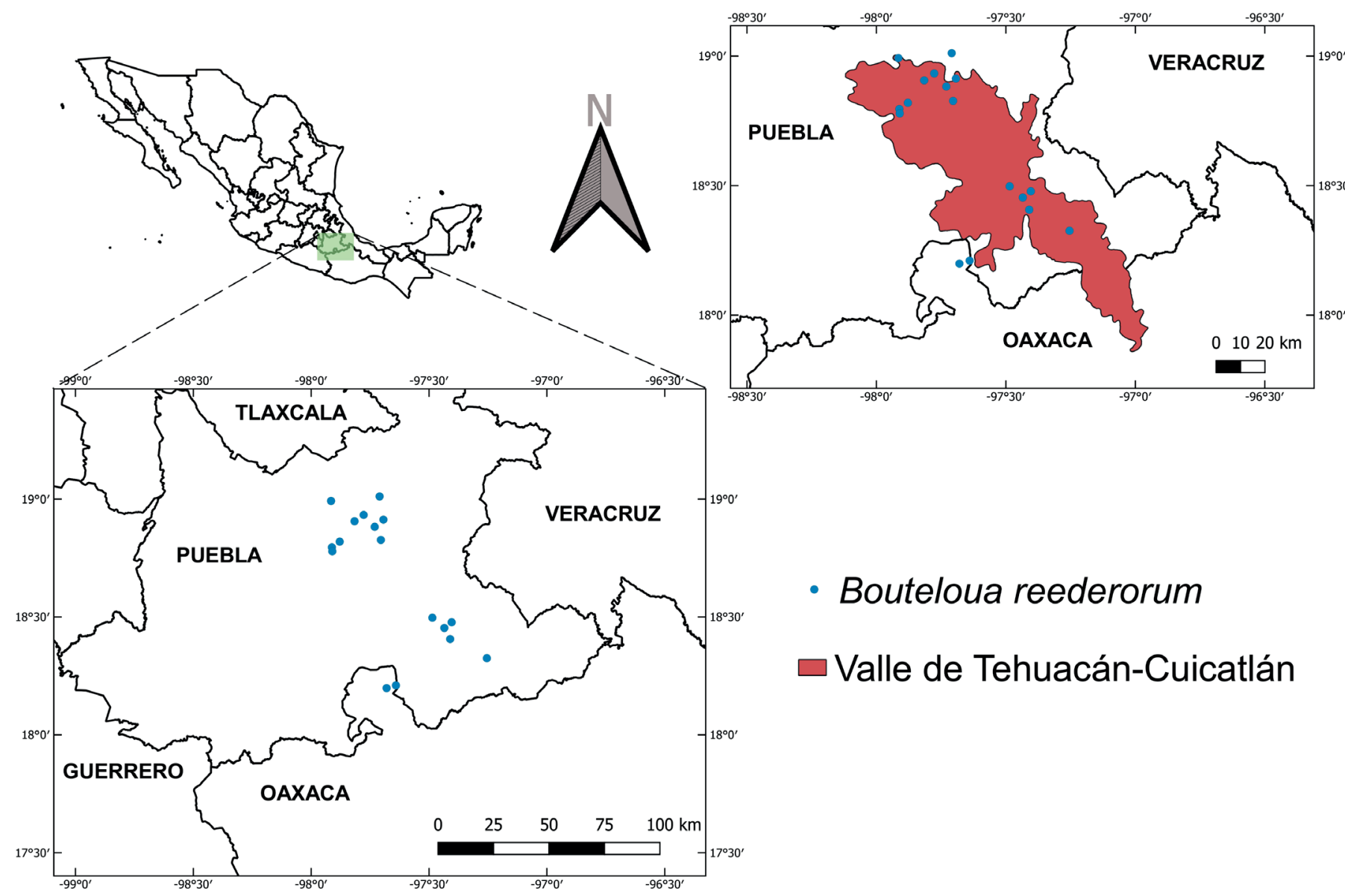

Figura 6: Distribución de Bouteloua reederorum Columbus en el Valle de Tehuacán-Cuicatlán, México.

El Salado, $2300 \mathrm{~m}, 18^{\circ} 52^{\prime} 57^{\prime \prime} \mathrm{N}, 97^{\circ} 43^{\prime} 49^{\prime \prime} \mathrm{W}, 23 . \mathrm{IX} .1983, A$. Sánchez y F. Hernández 54 (HUAP); extremo $\mathrm{S}$ de Santiago Alseseca, $2024 \mathrm{~m}, 18^{\circ} 49^{\prime} 36.8^{\prime \prime} \mathrm{N}, 97^{\circ} 42^{\prime} 14.9^{\prime \prime} \mathrm{W}, 6 . X .2018$, M. H. Sandoval 1033 (HUAA). Municipio Tehuacán, al S de la colonia Resurrección, orilla de la autopista Tehuacán Oaxaca, 1738 m, 18²7'12.8"N, 97²6'05.6"W, 6.X.2018, M. H. Sandoval 1034 (HUAA). Municipio Tepeyahualco de Cuauhtémoc, extremo $\mathrm{N}$ de la colonia Emiliano Zapata, 1935 m, 1849'10.8'"N, 9752'43.7"W, 6.X.2018, M. H. Sandoval 1031 (HUAA). Municipio Tlacotepec de Benito Juárez, 21 mi NW of Tehuacán, 1950 m, 12.IX.1963, J. R. Reeder y C. G. Reeder 3735 (ARIZ). Oaxaca, distrito Huajuapan, municipio Santiago Chazumba, extremo N de Santiago Chazumba, $1774 \mathrm{~m}, 18^{\circ} 11^{\prime} 54.4^{\prime \prime} \mathrm{N}, 97^{\circ} 40^{\prime} 47.4^{\prime \prime} \mathrm{W}, 14 . \mathrm{VIII.2019,}$ M. H. Sandoval 1052 (HUAA); límite estatal Oaxaca - Puebla, carretera 125, $2008 \mathrm{~m}, 18^{\circ} 12^{\prime} 35.4^{\prime \prime} \mathrm{N}, 97^{\circ} 38^{\prime} 24.6^{\prime \prime} \mathrm{W}$, 14.VIII.2019, M. H. Sandoval 1053 (HUAA).

\section{Discusión}

Bouteloua reederorum forma parte de la sección Triplathera (Endl.) P.M. Peterson, Romasch. \& Y. Herrera, junto con B. nervata Swallen, B. megapotamica (Spreng.) Kuntze y B. rigidiseta (Steud.) Hitchc. (Peterson et al., 2015). Este grupo incluye dos taxones con flósculos unisexuales y distribución restringida al centro del país (B. nervata y B. reederorum) y dos con flósculos bisexuales; uno de los cuales habita en el noreste de México y sureste de Estados Unidos de América (B. rigidiseta) y el otro es endémico de Sudamérica (B. megapotamica).

Aunque las relaciones filogenéticas de Bouteloua fueron estudiadas por Columbus et al. (1998) y Peterson et al. (2015), Scribner (1896) y Reeder et al. (1965) observaron similitud de ciertos caracteres morfológicos entre algunas especies de la sección Triplathera y sugirieron una posible relación entre ellos, aun cuando los consideraban géneros distintos. 


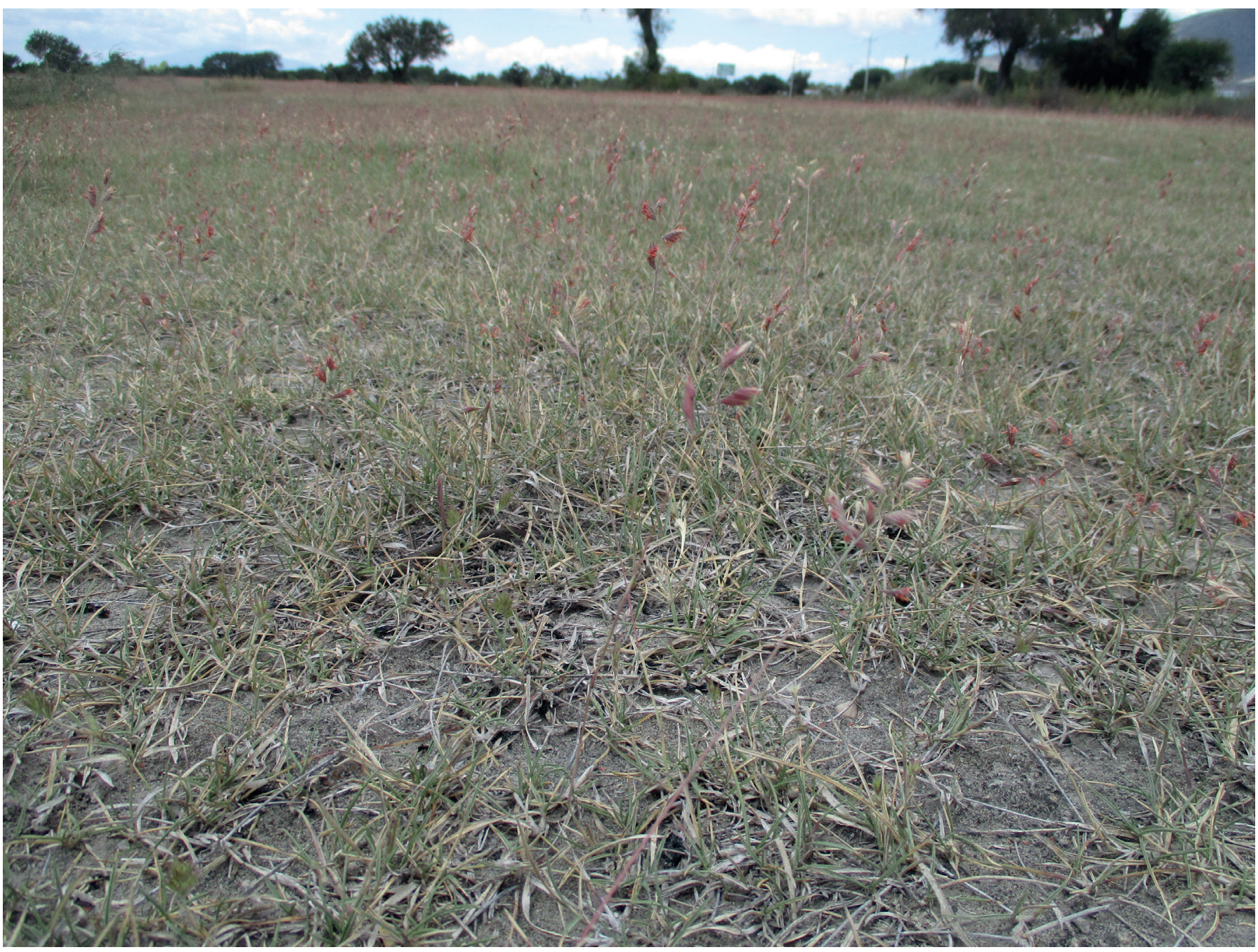

Figura 7: Pastizal de Bouteloua reederorum Columbus en Santiago Alseseca, Puebla, México.

Bouteloua nervata se incluyó dentro del género Buchlomimus Reeder, C. Reeder \& Rzed. por ser dioica (Reeder et al., 1965; Kinney et al., 2007). No obstante, Reeder et al. (1965) propusieron la combinación Buchlomimus nervatus (Swallen) Reeder, C. Reeder \& Rzed., y reconocieron que era morfológicamente similar a Bouteloua reederorum (= Pringleochloa stolonifera) y que ambas podrían estar cercanamente relacionadas. Por otro lado, en la descripción de Pringleochloa stolonifera (= Bouteloua reederorum), Scribner (1896) mencionó que Pringleochloa estaba cercanamente relacionado con Bouteloua, pero poseía una serie de caracteres que la diferenciaban, entre ellos el ser monoico y tener espigas femeninas en la base del macollo cuyas espiguillas pistiladas concuerdan en algunos aspectos con las espiguillas de Eutriana multiseta Nees (= Bouteloua megapotamica (Spreng.) Kuntze), como son: los flósculos rudimentarios aristados y la morfología de la lema.

Tras la transferencia de especies de los géneros $\mathrm{Bu}$ chlomimus y Pringleochloa a Bouteloua, Columbus (1999) planteó nuevas combinaciones y nombres; en Pringleochloa stolonifera (= Atheropogon stolonifer) propuso a Bouteloua reederorum, mientras que para Cyclostachya stolonifera designó a Bouteloua stolonifera. Posteriormente, $C$. stolonifera se consideró erróneamente como sinónimo de B. reederorum (Peterson et al., 2001), posiblemente debido a una confusión entre los nombres Pringleochola stolonifera y C. stolonifera, ya que ambos poseen el mismo epíteto específico.

La historia nomenclatural de $B$. reederorum es compleja, pero tras el redescubrimiento del holotipo del basó- 
nimo Atheropogon stolonifer (Liebmann 588) (SandovalOrtega et al., 2019), se corroboró que corresponde a un ejemplar pistilado de $B$. reederorum, por lo que los nombres Cathestecum stoloniferum (E. Fourn.) Griffiths (combinación para A. stolonifer (Griffiths, 1912)) y Cyclostachya stolonifera se descartan como sinónimos de $B$. reederorum.

Aunque Bouteloua reederorum podría ser una especie dioica (Columbus et al., 1998; Kinney et al., 2007), no hay suficiente evidencia para asegurarlo y es probable que en los ejemplares que presentan solo inflorescencias pistiladas o estaminadas, no han madurado las del sexo opuesto. En las colectas en campo se observó que un solo individuo de esta especie puede cubrir grandes superficies con estolones y presentar en algunos macollos inflorescencias de un solo sexo, mientras que, en otras porciones de la planta, se desarrollan inflorescencias del sexo contrario o ambas.

En las descripciones de Scribner (1896) y Beetle et al. (1999) no se mencionó la presencia de flósculos rudimentarios en las espiguillas masculinas de $B$. reederorum y se reportan por primera vez en este estudio, aunque son poco frecuentes. Se encontraron únicamente dos flósculos, ambos en espiguillas estériles de las ramas terminales en dos ejemplares distintos (M. H. Sandoval 1029 y 1032), que representan cerca de $6 \%$ del material colectado (32 individuos, correspondientes a nueve colectas y sus duplicados), por esta razón no se incluyeron en la descripción morfológica.

La mayoría de los registros (14 ejemplares) de $B$. reederorum, pertenecen al estado de Puebla, mientras que para Oaxaca son pocos (tres ejemplares), uno es el ejemplar tipo (Liebmann 588), cuya localidad es poco precisa (La Parada, Sierra de Oaxaca). García-Mendoza y Meave (2012) reportan esta especie en el estado y citan un ejemplar depositado en US (Hitchcock 6238); sin embargo, corresponde a Bouteloua varia (Swallen) Columbus (= Cathestecum varium Swallen). Pacheco-Rivera y Dávila-Aranda (2004), registraron a $B$. reederorum en el distrito de Huajuapan y aunque no mencionaron colectas o localidades, se realizaron exploraciones en dicha área y se localizaron poblaciones en dos localidades del municipio Santiago Chazumba, muy cerca del límite con Puebla.

\section{Conclusiones}

Bouteloua reederorum es una especie restringida al Valle de Tehuacán-Cuicatlán, en el sur de México y difiere de $B$. stolonifera (= Cyclostachya stolonifera), la cual también es endémica del país, pero con distribución más amplia, desde Ciudad de México hasta Durango. Ambos taxones pueden distinguirse por la ramificación de sus inflorescencias, forma de la lígula, proporción de sexos y su distribución geográfica. En raras ocasiones se pueden presentar flósculos rudimentarios en algunas espiguillas masculinas de $B$. reederorum. Es necesario realizar estudios fenológicos que demuestren si B. reederorum puede ser dioica.

\section{Contribución de autores}

MHSO, MESD, RCT y EPMB concibieron y diseñaron el estudio. MHSO, MESD y RCT realizaron colectas. MHSO identificó ejemplares, elaboró el manuscrito y las figuras. MESD, RCT y EPMB revisaron el manuscrito. Todos los autores contribuyeron a la discusión y aprobación del manuscrito final.

\section{Financiamiento}

Este estudio fue apoyado por el Consejo Nacional de Ciencia y Tecnología a MHSO (número de beca 88032).

\section{Agradecimientos}

A Guillermo Cabral por su ayuda durante el trabajo de campo. A George M. Ferguson, del herbario de la Universidad de Arizona por facilitar los ejemplares.

\section{Literatura citada}

Beetle, A. A., A. Bolaños M., J. A. Miranda S., L. Aragón M., M. A. Vergara B., A. Chimal H., M. M. Castillo B., O. M. Galván G., J. L. Villalpando P., M. Lizama, J. Valdés R., E. Manrique de S. y A. M. Rodríguez R. 1999. Las Gramíneas de México. Tomo V. Secretaría de Agricultura, Ganadería y Desarrollo Rural, Comisión Técnico Consultiva de Coeficientes de Agostadero. México, D.F., México. 482 pp.

Columbus, J. T. 1999. An expanded circumscription of Bouteloua (Gramineae: Chloridoideae): new combinations and names. Aliso 18(1): 61-65. DOI: https://doi.org/10.5642/ aliso.19991801.16

Columbus, J. T., M. S. Kinney, R. Pant y M. E Siqueiros-Delgado. 1998. Cladistic parsimony analysis of internal transcribed 
spacer region (nrDNA) sequences of Bouteloua and relatives (Gramineae: Chloridoideae). Aliso 17(2): 99-130. DOI: https://doi.org/10.5642/aliso.19981702.03

Dávila, P., M. T. Mejia-Saulés, A. M. Soriano-Martínez y Y. HerreraArrieta. 2018. Conocimiento taxonómico de la familia Poaceae en México. Botanical Sciences 96(3): 462-514. DOI: https://doi.org/10.17129/botsci.1894

de la Cerda-Lemus, M. 1996. Las gramíneas de Aguascalientes. Universidad Autónoma de Aguascalientes. Aguascalientes, México. 212 pp.

DMA. 1991. Technical Report 8350.2: World Geodetic System 1984, Its Definition and Relationships with Local Geodetic Systems. 2nd ed. The Defense Mapping Agency (DMA). Springfield, USA. 169 pp.

Engelmann, G. 1986. Instructions for the collection and preservation of botanical specimens. Annals of the Missouri Botanical Garden 73: 504-507.

Espejo-Serna, A. y A. R. López-Ferrari. 2000. Las Monocotiledóneas Mexicanas, Partes IX a XI (Pandanaceae a Zosteraceae). Consejo Nacional de la Flora de México, Universidad Autónoma Metropolitana, Comisión Nacional para el Conocimiento y Uso de la Biodiversidad (CONABIO). México, D.F., México. 337 pp.

García-Mendoza, A. J. y J. A. Meave. 2012. Diversidad florística de Oaxaca: de musgos a angiospermas. Universidad Nacional Autónoma de México, Comisión Nacional para el Conocimiento y Uso de la Biodiversidad (CONABIO), Instituto Estatal de Ecología y Desarrollo Sustentable de Oaxaca. México, D.F., México. 351 pp.

Gould, F. W. 1979. The genus Bouteloua (Poaceae). Annals of the Missouri Botanical garden 66 (3): 348-416. DOI: https://doi. $\operatorname{org} / 10.2307 / 2398834$

Griffiths, D. 1912. The grama grasses: Bouteloua and related genera. Contributions from the United States National Herbarium 14(3): 343-428.

Herrera-Arrieta, Y. 2001. Las gramíneas de Durango. Centro Interdisciplinario de Investigación para el Desarrollo Integral Regional (CIIDIR), Comisión Nacional para el Conocimiento y Uso de la Biodiversidad (CONABIO). Durango, México. 478 pp.

Herrera-Arrieta, Y. 2014. Additions and updated names for grasses of Durango, Mexico. Acta Botanica Mexicana 106: 79-95. DOI: https://doi.org/10.21829/abm106.2014.214
Herrera-Arrieta, Y. y P. M. Peterson. 2018. Grasses of Chihuahua, Mexico. Smithsonian Contributions to Botany 107: 1-380. DOI: https://doi.org/10.5479/si.1938-2812.107

Herrera-Arrieta, Y., P. M. Peterson y A. Cortés-Ortiz. 2010. Gramíneas de Zacatecas, México. Comisión Nacional para el Conocimiento y Uso de la Biodiversidad (CONABIO), Instituto Politécnico Nacional (IPN), Smithsonian Institution. Texas, EUA. 239 pp.

Herrera-Arrieta, P. M. Peterson y M. de la Cerda-Lemus. 2004. Revisión de Bouteloua Lag. (Poaceae). Centro Interdisciplinario de Investigación para el Desarrollo Integral Regional (CIIDIR), Comisión Nacional para el Conocimiento y Uso de la Biodiversidad (CONABIO), Filo de Agua. Durango, México. 187 pp.

JSTOR. 2019. Global Plants. https://plants.jstor.org/ (consultado noviembre de 2019).

Kinney, M. S., J. T. Columbus y E. A. Friar. 2007. Dicliny in Bouteloua (Poaceae: Chloridoideae): Implications for the evolution of dioecy. Aliso 23(1): 605-614. DOI: https://doi.org/10.5642/ aliso. 20072301.45

P. 2019. Muséum national d'Histoire naturelle, Vascular Plants. Paris, France. https://science.mnhn.fr/institution/mnhn/ collection/p/list (consultado noviembre de 2019).

Pacheco-Rivera, D. y P. Dávila-Aranda. 2004. Sinopsis de las gramíneas de Oaxaca, México. Acta Botanica Mexicana 69: 83114. DOI: https://doi.org/10.21829/abm69.2004.982

Peterson, P. M., K. Romaschenko y Y. Herrera-Arrieta. 2015. Phylogeny and subgeneric classification of Bouteloua with a new species, $B$. herrera-arrietae (Poaceae: Chloridoideae: Cynodonteae: Boutelouinae). Journal of Systematics and Evolution 53(4): 351-366. DOI: https://doi.org/10.1111/ jse.12159

Peterson, P. M., R. J. Soreng, G. Davidse, T. S. Filgueiras, F. O. Zuloaga, E. J. Judziewicz y R. J. Soreng. 2001. Catalogue of New World Grasses (Poaceae): II. Subfamily Chloridoideae. Contributions from the United States National Herbarium 41: 1-255.

QGIS Development Team. 2017. Quantum GIS Geographic Information System. Quantum GIS Development Team. Open Source Geospatial Foundation Project. Vienna, Austria.

Reeder, J. R. 1969. Las gramíneas dioicas de México. Boletín de la Sociedad Botánica de México 30: 121-126. DOI: https://doi. org/10.17129/botsci.1102

Reeder, J. R. y C. G. Reeder. 1963. Notes on Mexican grasses II. Cyclostachya, a new dioecious genus. Bulletin of 
the Torrey Botanical Club 90: 193-2001. DOI: https://doi. $\operatorname{org} / 10.2307 / 2482754$

Reeder, J. R., C. G. Reeder y J. Rzedowski. 1965. Notes on Mexican Grasses III. Buchlomimus, another dioecious genus. Brittonia 17(1): 26. DOI: https://doi.org/10.2307/2805389

Sánchez-Ken, J. G. 2019. Riqueza de especies, clasificación y listado de las gramíneas (Poaceae) de México. Acta Botanica Mexicana 126: e1379. DOI: https://doi.org/10.21829/ abm126.2019.1379

Sandoval-Ortega, M. H., M. E. Siqueiros-Delgado, E. PérezMolphe-Balch y R. Cerros-Tlatilpa. 2019. The holotype of Atheropogon stolonifer (=Bouteloua reederorum) and lectotypification of the name Triathera juncea (=B. juncea) (Poaceae, Chloridoideae). Phytotaxa 415(1): 79-84. DOI: https://doi.org/10.11646/phytotaxa.415.1.7
Scribner, L. F. 1896. New North American Grasses. Botanical Gazette 21(3): 133-139. DOI: https://doi.org/10.1086/327314

Thiers, B. 2019+. Index Herbariorum. A global directory of public herbaria and associated staff. New York Botanical Garden's Virtual Herbarium. http://sweetgum.nybg.org/science/ih (consultado junio de 2019).

TROPICOS. 2020. Tropicos.org. http://www.tropicos.org/ (consultado marzo de 2020).

US. 2019. Botany Collection, National Museum of Natural History. Smithsonian Institution. https://collections.nmnh.si.edu/ search/botany/ (consultado diciembre de 2019).

Villaseñor, J. L. 2016. Checklist of the native vascular plants of Mexico. Revista Mexicana de Biodiversidad 87: 559-902. DOI: https://doi.org//10.1016/j.rmb.2016.06.017 